\title{
Variants of GRM7 as risk factor and response to antipsychotic therapy in schizophrenia
}

Wei Liang ${ }^{1,2}$, Hao Yu $\mathbb{0}^{3}$, Yi Su ${ }^{1,2}$, Tianlan Lu ${ }^{1,2}$, Hao Yan $\mathbb{D}^{1,2}$, Weihua Yue $\mathbb{B}^{1,2,4,5}$ and Dai Zhang ${ }^{1,2,4}$

\begin{abstract}
Genome-wide association study (GWAS) has determined the metabotropic glutamate receptor 7 (GRM7) gene as potential locus for schizophrenia risk variants; However, the relationship between the GRM7 variants and the risk of schizophrenia is still uncertain, and there are significant individual variations in response to the antipsychotic drugs. In order to identify susceptible gene and drug-response-related markers, 2413 subjects in our research were chosen for determining drug-response-related markers in schizophrenia. The rs1516569 variant $\left(\mathrm{OR}=0.95, P<3.47 \times 10^{-4}\right)$ was a significant risk factor, and a single-nucleotide polymorphism of GRM7 gene- rs9883258 (OR $\left.=0.84, P=2.18 \times 10^{-3}\right)$ has been determined as potential biomarkers for therapeutic responses of seven commonly used antipsychotic drugs (aripiprazole, haloperidol, olanzapine, perphenazine, quetiapine, risperidone and ziprasidone) in Chinese Han population; Significant associations with treatment response for several single-nucleotide polymorphisms in every antipsychotic drugs, such as rs779746 (OR $=1.39, P=0.03)$, rs480409 (OR=0.73, $P=0.04)$, rs78137319 $(\mathrm{OR}=3.09, P=$ 0.04), rs $1154370(\mathrm{OR}=1.51, P=0.006)$ have been identified in our study. Hence our research elucidates that GRM7 variants play the critical role of predicting the risk of schizophrenia and antipsychotic effect of seven common drugs.
\end{abstract}

\section{Introduction}

Schizophrenia (SCZ) is a debilitating psychiatric syndrome with decreased quality of life and shortened life expectancy, which affects around $1 \%$ of people at some point in their life ${ }^{1,2}$. Although the etiology of SCZ is still unknown, twin investigations have elucidated that it is highly inheritable neuropsychiatry disorder ${ }^{3}$. Probing into genetic mechanisms of SCZ has appealed to more and more concern.

Recently, SZDB (www.szdb.org) have identified hundreds of loci displaying a genome-wide significant correlation with SCZ in diverse population ${ }^{4}$. Of these risk loci, some are related major hypotheses of the etiology and treatment of SCZ, such as genes involved in glutamatergic

\footnotetext{
Correspondence: Weihua Yue (dryue@bjmu.edu.cn) or

Dai Zhang (daizhang@bjmu.edu.cn)

${ }^{1}$ Institute of Mental Health, Peking University Sixth Hospital, 100191 Beijing,

China

${ }^{2} \mathrm{NHC}$ Key Laboratory of Mental Health, National Clinical Research Center for Mental Disorders and Key Laboratory of Mental Health, Ministry of Health (Peking University Sixth Hospital), 100191 Beijing, China

Full list of author information is available at the end of the article.
}

(GRM3, GRIN2A, GRIA1) neurotransmission ${ }^{5}$. However, the roles of other glutamatergic genes in SCZ was still unclear.

One of the major hypotheses of SCZ is the glutamatergic neuronal dysfunction hypothesis ${ }^{6}$. Some single nucleotide polymorphisms (SNPs) of metabotropic glutamate receptor 7 (GRM7) have also been found to be related to SCZ in different populations ${ }^{7-9}$. GRM7 is considered to be a critical actor in regulating synaptic glutamate transmission and $\gamma$ amino butyric acid (GABA) release in the central nervous system $^{9-11}$. Whereas, though previous investigations have strongly revealed GRM7 as the SCZ risk factor, these studies only focus on a small amount of SNPs in limited sample. To expand the knowledge of GRM7 in SCZ susceptibility, researches with large sample data and with broad analysis are needed.

On the other hand, evidence implies that genetic variants of GRM7 play a critical part in different antipsychotic drug response $^{12-14}$. Nowadays, the Pharmacotherapy is the main way to improve the symptoms of schizophrenia ${ }^{15}$. According to the pharmacological research, the subjects 
have different personalized response to the seven common anti-antipsychotic drugs.It is necessary to determine the drug-efficient markers in order to predict the curative effect. Genetic predictors discovered by pharmacogenetic investigation could provide a clue for clinicians to select the reasonable therapy for SCZ patients. The previous researches have illustrated positive associations between genetic variation and efficacy of antipsychotic drugs treatment ${ }^{16,17}$. However, these studies mainly center at the European populations or include small samples. Therefore, these results need to be confirmed in diffident population and validated in concrete clinical application.

In this investigation, we employed GWAS samples from diverse populations to analyze a high density of SNPs in GRM7 gene. The discovered SCZ risk SNPs were then detected for their correlation with GRM7 expression. We hypothesized that GRM7 might contribute to the treatment outcomes of antipsychotic drugs; therefore, we examined the associations between the GRM7 genotype and treatment response of antipsychotic drug.

\section{Materials and methods SCZ GWAS data}

We meta-analyzed GRM7 associations across three GWAS datasets in diverse populations, via a fixed-effects model with inverse-variance weighting. The GWAS datasets were described as follows. Concrete information concerning sample description, diagnosis, genotyping and statistical analyses can be discovered in the original research $^{18-20}$.

Asian sample: we used the GWAS data from the Chinese Schizophrenia Collaboration Group $(\mathrm{CSCG})^{19}$ and Asian project of Psychiatric Genomics Consortium (PGCAsian) ${ }^{20}$. The SCZ GWAS of CSCG is a meta-analysis of four independent schizophrenia GWAS datasets (GWAS1-GWAS4) were conducted, including a total of 4565 patients and 5947 controls $^{19}$. All Han Chinese samples included were acquired from plentiful cooperating hospitals in the CSCG. Genotyping of the samples was conducted using Illumina (San Diego, CA, USA). Meanwhile, the PGC-Asian GWAS compiled 22,778 SCZ patients and 35,362 controls from 20 samples in Singapore, Japan, Indonesia, Korea, Hong Kong, Taiwan, and mainland $\mathrm{China}^{20}$. Meta-analyses across all PGC-Asian samples were conducted using a fixed-effects model with inverse-variance weighting. After removing sample overlap between CSCG and PGC-Asian (e.g., GWAS3 and GWAS4 of CSCG), 25,119 SCZ patients and 38,408 controls were used for the analysis.

European sample: The SCZ GWAS of European sample was from a large-scale SCZ GWAS of the PGC and CLOZUK study ${ }^{18}$. After excluding related and overlapping samples of PGC and CLOZUK study, it comprised of 40,675 cases and 64,643 controls. The SNP associations of
GRM7 gene were download from the website (http:// walters.psycm.cf.ac.uk).

We performed a meta-analysis of the SNPs covering the GRM7 region in all samples with PLINK v1.90, and used odds ratio (OR) and standard error (SE) for the estimation of heterogeneity between individual samples. The metaanalysis was performed using the classical inverse variance weighted methods. The regional association results of SNPs were plotted using LocusZoom ${ }^{21}$. After meta-analysis, we performed Linkage disequilibrium (LD)-based clumping with a SNP window of $500 \mathrm{~kb}$ and $r^{2}=0.1$.

\section{Pharmacogenetics study of GRM7 gene Participants}

To examine the associations between GRM7 variations and treatment response in patients with $\mathrm{SCZ}$, we performed a pharmacogenetics study using our previous data $^{22}$. Briefly, all subjects were of Chinese Han ethnicity, and were born and residing in China. The consensus diagnoses were made by at least two experienced senior psychiatrists according to the Diagnosis and Statistic Manual of Mental Disorders, 4th edition (DSM-IV) criteria for schizophrenia. Patients with previously diagnosed diabetes, thyroid disease, hypertension, heart disease and other severe physical diseases were excluded. Patients who met inclusion criteria were randomly assigned (1:1:1:1:1:1:1) to seven groups (olanzapine, risperidone, quetiapine, aripiprazole, ziprasidone, and haloperidol or perphenazine) for six-week treatment.

Clinical effect was assessed based on the Positive and Negative Syndrome Scale (PANSS), which include the positive, negative and general psychopathology subscales. The patients visited the participating clinicians at weeks 2, 4 , and 6 , and their PANSS scores were then recorded. For each patient, the PANSS reduction rate was defined as $100 \times$ (PANSS score at endpoint-PANSS score at baseline)/ (PANSS score at baseline -30). Different from our previous study $^{22}$, the antipsychotic response was classed into two groups, clinical good responders were defined as patients with 50\% or even higher reduction in PANSS scores; poor responders were defined as patients with lower reduction than $50 \%$ in PANSS scores. A detailed description of the participants can be found in our previous study ${ }^{22}$. The study was approved by the research ethics committees and institutional review boards of each local hospital. All procedures were conducted in accordance with the principles expressed in the Declaration of Helsinki. The study was registered under the clinical trial number ChiCTR-TRC10000934 (http://www.chictr.org/).

\section{Genotyping}

Genomic DNA was extracted using the QIAamp DNA Mini Kit (QIAGEN, Hilden, Germany). Sample genotype sequencing was genotyped with Illumina Human Omni 
Table 1 Descriptive statistics for subjects in meta-analysis.

\begin{tabular}{|c|c|c|c|c|c|c|}
\hline \multirow[t]{2}{*}{ Analyses } & \multicolumn{3}{|l|}{ Cases } & \multicolumn{3}{|l|}{ Controls } \\
\hline & Sample size & Mean age (s.d.) & Male/female & Sample size & Mean age (s.d.) & Male/female \\
\hline GWAS1 & 746 & $34.5(8.7)$ & $396 / 350$ & 1599 & $35.8(7.8)$ & $846 / 753$ \\
\hline GWAS2 & 1595 & 30.1 (10.7) & $859 / 736$ & 1447 & 29.7 (9.8) & $350 / 1090$ \\
\hline
\end{tabular}

s.d. standard deviation.

ZhongHua-8 Beadchips (Illumina, San Diego, CA, USA). Genotype imputation for the discovery sample. Genotype imputation was done with the pre-phasing imputation stepwise approach implemented in IMPUTE2 and SHAPEIT (version 2.r727) ${ }^{17,21}$. Haplotypes derived from phase I of the 1000 Genomes Project (release version 3) were used as references. SNPs with imputation quality scores below a set threshold (info score $<0.9$ ) were excluded from further analyses. All genomic locations are given as National Center for Biotechnology Information Build 37 coordinates. We extracted the GRM7 genotype from the imputed data. Totally, 652 SNPs in GRM7 were extracted for this study. After LD-based pruning (a window of 500 SNPs and $\left.r^{2}=0.1\right), 19$ SNPs were retained for the pharmacogenetics study.

\section{Statistical analysis}

Cohort characteristics including gender, age and weight differences of demographic and clinical variables were examined first to affirm the homogeneity of good responders and poor responders in our samples. We used logistic regression under an additive genetic model to evaluate the associations between the allele dosages and the PANSS percentage change values in PLINK v1.90 23 . The $P$ values were adjusted by Bonferroni correction.

\section{Gene expression analysis}

We examined cis-eQTL data of GRM7 gene in the Brain Expression Consortium (BRAINEAC, http://caprica. genetics.kcl.ac.uk/BRAINEAC/), which consisted of 134 neuropathologically normal donors from the MRC Sudden Death Brain Bank in Edinburgh and Sun Health Research Institute, the gene expression was profiled on the Affymetrix Exon 1.0 ST array ${ }^{24}$. In BRAINEAC database, we can examine generated eQTL data for ten human brain regions (cerebellar cortex, frontal cortex, hippocampus, inferior olivary nucleus, occipital cortex, putamen, substantia nigra, temporal cortex, thalamus and intralobular white matter $)^{24}$. To explore whether the GRM7 gene is differentially expressed in SCZ patients compared to controls, we obtained publicly available expression data from BrainSeq database ${ }^{25}$. The BrainSeq project performed RNA-seq analysis using DLPFC of
412 subjects (175 patients with SCZ and 237 unaffected controls) ${ }^{25}$.

\section{Results}

\section{GRM7 and the risk of SCZ}

Demographic and descriptive data are listed in Table 1. The association data of 3273 SNPs spanning the GRM7 region were acquired from both European and Asian GWAS samples, consisted of 65,794 cases and 103,051 controls. Then, a meta-analysis of these SNPs including all samples (Fig. 1a) was carried out. After LD-based clumping, 79 independent SNPs were retained. Only the SNP $\operatorname{rs} 1516569\left(\mathrm{OR}=0.95, P<3.47 \times 10^{-4}\right.$, Table 2$)$ was significantly associated with SCZ after Bonferroni correction $\left(P<0.05 / 79=6.30 \times 10^{-4}\right)$. However, the SNP rs1516569 is still far from genome-wide significance $\left(P<5 \times 10^{-8}\right)$. To examine whether rs1516569 also modulates the transcription and expression of GRM7, we employed the gene expression data in ten brain regions obtained from the BRAINEAC database. Interestingly, the risk allele $(G)$ of $\operatorname{rs} 1516569\left(P=1.0 \times 10^{-3}\right)$ was significantly correlated with higher GRM7 expression in putamen region (Fig. 1b). Furthermore, we performed differential expression analysis of GRM7 gene using BrainSeq database ${ }^{25}$. Compared with controls, the expression level of GRM7 transcript was significantly higher in SCZ patients ( $t=3.19, P=1.55 \times 10^{-3}$; Fig. 1c). These results suggested that the risk SNP probably was conducive to the disease risk by modulating GRM7 expression of brain.

\section{GRM7 and treatment response of antipsychotic drugs}

Demographic and descriptive data are listed in Table 3. Based on the assumption that genetic moderators of response are similar across antipsychotics, we first performed a combined analysis across seven antipsychotic drugs. Among 19 LD-independent SNPs, the SNP rs9883258 showed vital correlation with treatment responses to antipsychotic drugs $(\mathrm{OR}=0.84, P=2.18 \times$ $10^{-3}$; Table 4). The result remains significant after Bonferroni correction $\left(P<2.63 \times 10^{-3}\right.$, i.e., $\left.0.05 / 19\right)$.

Then, we performed a second investigation in patients treated with specific antipsychotic drugs. Several SNPs were found to be nominally with several specific 


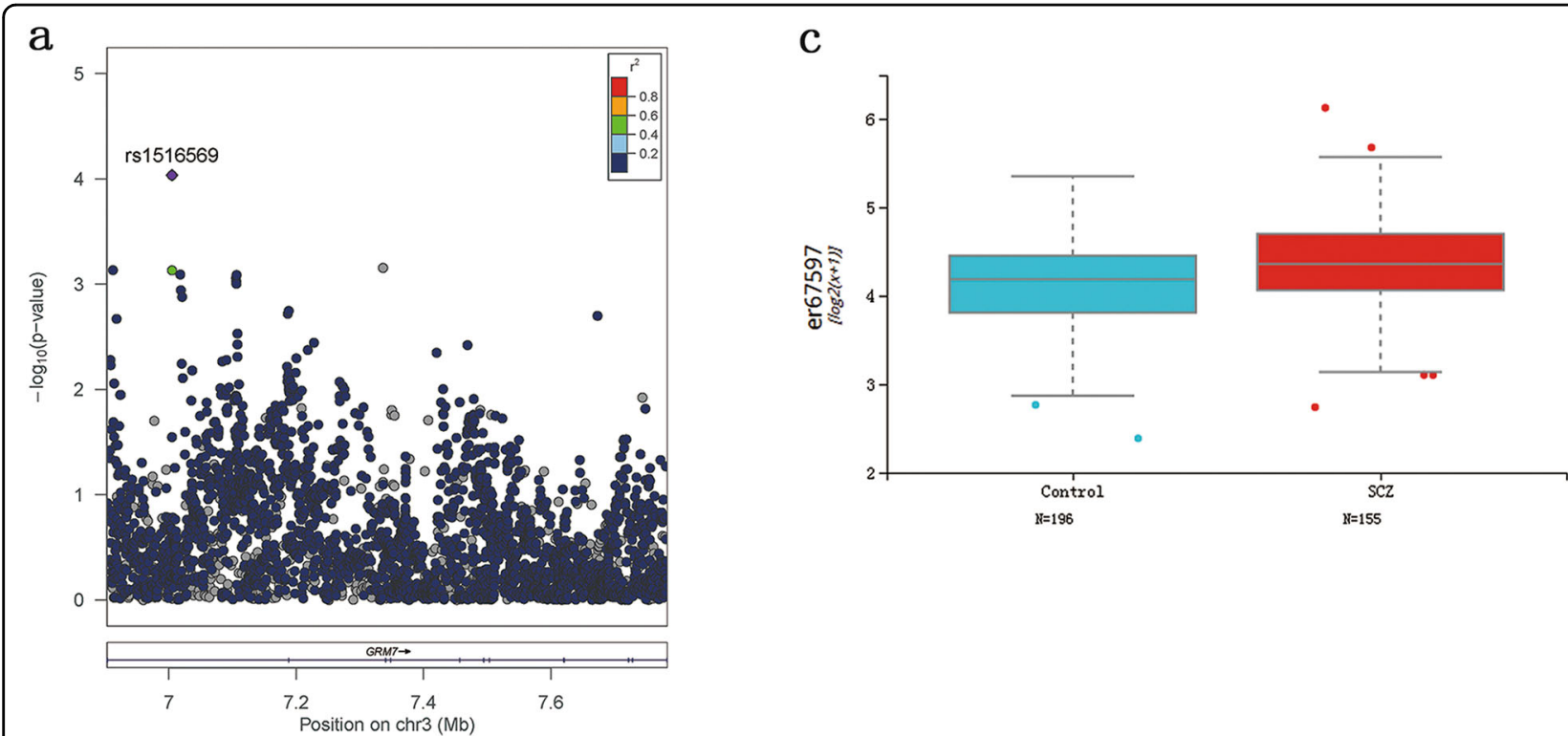

b Affymetrix ID 2609210 stratified by rs1516569 (chr3: 7004873)

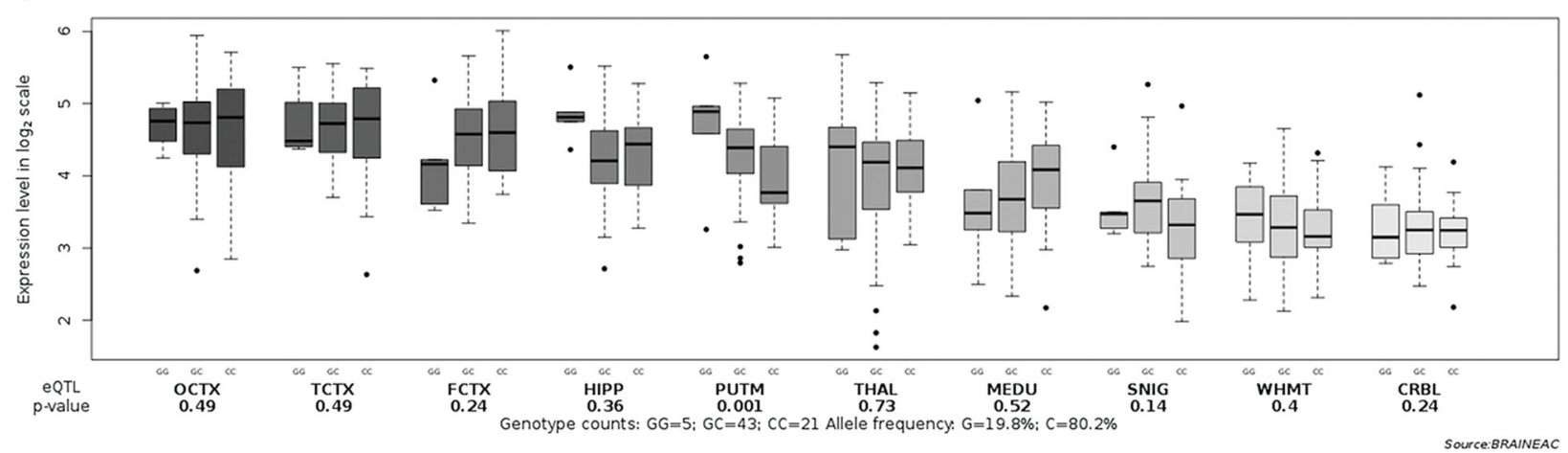

Fig. 1 Association between the SNP rs1516569 of GRM7 and SCZ risk. a Genetic association of GRM7 SNPs with risk for SCZ in meta-analysis of three ethnic samples; b) eQTL analysis of the SNP rs1516569 in BRAINEAC database (http://caprica.genetics.kcl.ac.uk/BRAINEAC/24; ${ }^{24}$ ) GRM7 gene is differentially expressed in SCZ patients compared to controls in BrainSeq databse (http://eqtl.brainseq.org/) ${ }^{25}$.

Table 2 The associations between the SNP rs1516569 of GRM7 and schizophrenia.

\begin{tabular}{|c|c|c|c|c|c|c|c|}
\hline Study & Chr & BP & SNP & A1 & A2 & OR & $\mathbf{P}$ \\
\hline GWAS1 of $\operatorname{CSCG}^{19}$ (746 cases and 1599 controls) & 3 & 7004873 & rs1516569 & C & G & 1.06 & 0.66 \\
\hline GWAS2 of CSCG $^{19}$ (1595 cases and 1447 controls) & 3 & 7004873 & rs1516569 & C & G & 1.11 & 0.12 \\
\hline PGC-Asian ${ }^{20}$ (22,778 cases and 35,362 controls) & 3 & 7004873 & rs1516569 & C & G & 0.97 & 0.72 \\
\hline PGC-Clozuk ${ }^{18}$ (40,675 cases and 64,643 controls) & 3 & 7004873 & rs1516569 & C & G & 0.95 & 7.63E-05 \\
\hline Meta-analysis 65,794 cases and 103,051 controls & 3 & 7004873 & rs1516569 & C & G & 0.85 & 3.47E-04 \\
\hline
\end{tabular}

GWAS1 and GWAS2 of CSCG were not included in PGC Asian project. Therefore, we performed a meta-analysis of four GWAS datasets.

CHR chromosome, SNP single nucleotide polymorphism, BP base position, A1/A2 minor allele/major allele, OR odds ratio.

antipsychotic drug (Supplementary Tables $1-7)$. In olanzapine group, the SNPs rs779746 $(\mathrm{OR}=1.39, P=0.03$; Supplementary Table 1) and rs480409 $(\mathrm{OR}=0.73$, $P=0.04$; Supplementary Table 1 ) were nominally associated with treatment response. The SNP rs78137319 $(\mathrm{OR}=3.09, P=0.04$; Supplementary Table 2$)$ was nominally associated with treatment response of quetiapine. The SNP rs1154370 was nominally associated with 
Table 3 Descriptive statistics for antipsychotic patientrelated variables.

\begin{tabular}{lll}
\hline Variable & Poor responders & Good responders \\
\hline No. & 991 & 1422 \\
Male, No. (\%) & $498(50.3)$ & $738(51.9)$ \\
Female, No. (\%) & $493(49.7)$ & $684(48.1)$ \\
Age, mean (SD), y & $31.4(8.0)$ & $31.5(8.0)$ \\
Antipsychotic drugs & & \\
Olanzapine & 142 & 283 \\
Risperidone & 133 & 279 \\
Aripiprazole & 185 & 212 \\
Quetiapine & 172 & 214 \\
Ziprasidone & 199 & 209 \\
Haloperidol & 79 & 113 \\
Perphenazine & 81 & 112 \\
PANSS total score, mean & $(S D)$ & $12.5(16.0)$ \\
Baseline & $11.8(14.6)$ & $9.8(13.9)$ \\
6 week & $18.3(27.0)$ & \\
\hline
\end{tabular}

treatment response of risperidone $(\mathrm{OR}=1.51, P=0.006$; Supplementary Table 3) and haloperidol $(\mathrm{OR}=1.73$, $P=9.0 \times 10^{-3}$; Supplementary Table 4). The SNP rs779746 $(\mathrm{OR}=0.61, P=0.02$; Supplementary Table 5$)$ was nominally associated with treatment response of perphenazine.

\section{Discussion}

With regard to the core risk factors, the GRM7 gene is significantly correlated with the schizophrenia patients. Glutamatergic neurotransmission may participate in many respects of normal brain activity and be disturbed in the state of neuropathology. The metabotropic glutamate receptors include three different groups and GRM4, GRM6, GRM7, and GRM8 belong to Group III, Group II and III receptors play a role in inhibiting the cyclic AMP cascade $^{26}$. The gene of GRM7 include several transcript variants encoding various isoforms. Our meta-analysis showed that rs1516569 in GRM7 was significantly associated with SCZ; this favorable SNP has not been determined in two earlier Caucasian independent samples ${ }^{27,28}$ and in the two Chinese samples ${ }^{29,30}$. The 1000 Genomes Project Phase 3 database (http://grch37.ensembl.org) showed that there are significant differences in the minor

Table 4 Genotype and allele frequencies of significant SNPs associated with antipsychotic treatment.

\begin{tabular}{|c|c|c|c|c|c|c|c|c|c|c|c|}
\hline $\mathrm{CHR}$ & SNP & BP & A1 & A2 & F_A & F_U & OR & SE & L95 & U95 & $P$ \\
\hline 3 & rs9883258 & 7419156 & G & A & 0.4708 & 0.5156 & 0.84 & 0.06 & 0.75 & 0.94 & 0.00218 \\
\hline 3 & rs9882058 & 6937327 & C & $\mathrm{T}$ & 0.0116 & 0.0182 & 0.63 & 0.24 & 0.39 & 1.02 & 0.0578 \\
\hline 3 & rs7374553 & 7528503 & G & A & 0.4877 & 0.4617 & 1.11 & 0.06 & 0.99 & 1.25 & 0.0748 \\
\hline 3 & rs1154370 & 7062465 & G & $\mathrm{T}$ & 0.4863 & 0.4627 & 1.10 & 0.06 & 0.98 & 1.23 & 0.106 \\
\hline 3 & rs779746 & 7578531 & $C$ & $\mathrm{~T}$ & 0.4898 & 0.4662 & 1.10 & 0.06 & 0.98 & 1.23 & 0.1063 \\
\hline 3 & rs670764 & 7026341 & G & A & 0.4318 & 0.4092 & 1.10 & 0.06 & 0.98 & 1.23 & 0.1178 \\
\hline 3 & rs342026 & 6908535 & G & A & 0.4909 & 0.5111 & 0.92 & 0.06 & 0.82 & 1.03 & 0.1665 \\
\hline 3 & rs712767 & 7443688 & C & $\mathrm{T}$ & 0.4655 & 0.4521 & 1.06 & 0.06 & 0.94 & 1.19 & 0.3556 \\
\hline 3 & rs1504047 & 7748843 & $\mathrm{~T}$ & G & 0.2996 & 0.3118 & 0.94 & 0.06 & 0.83 & 1.07 & 0.3639 \\
\hline 3 & rs13321431 & 7001961 & $\mathrm{~T}$ & G & 0.4842 & 0.495 & 0.96 & 0.06 & 0.85 & 1.07 & 0.4612 \\
\hline 3 & rs480409 & 7010081 & C & $\mathrm{T}$ & 0.3952 & 0.3905 & 1.02 & 0.06 & 0.91 & 1.15 & 0.7421 \\
\hline 3 & rs78137319 & 7348332 & A & G & 0.01371 & 0.01261 & 1.09 & 0.26 & 0.66 & 1.80 & 0.7425 \\
\hline 3 & rs11918634 & 7683434 & A & G & 0.4205 & 0.4162 & 1.02 & 0.06 & 0.91 & 1.14 & 0.7664 \\
\hline 3 & rs74668910 & 7068619 & $\mathrm{~T}$ & G & 0.01055 & 0.01009 & 1.05 & 0.29 & 0.59 & 1.85 & 0.8772 \\
\hline 3 & rs2291867 & 7340164 & G & A & 0.4965 & 0.4985 & 0.99 & 0.06 & 0.88 & 1.11 & 0.8911 \\
\hline 3 & rs17047886 & 7780116 & C & $\mathrm{T}$ & 0.01372 & 0.01413 & 0.97 & 0.25 & 0.60 & 1.58 & 0.906 \\
\hline 3 & rs6778030 & 7153652 & G & A & 0.4662 & 0.4647 & 1.01 & 0.06 & 0.90 & 1.13 & 0.9147 \\
\hline 3 & rs7623046 & 7510856 & A & G & 0.4989 & 0.5 & 1.00 & 0.06 & 0.89 & 1.12 & 0.9425 \\
\hline 3 & rs3749380 & 6903297 & $\mathrm{~T}$ & C & 0.2827 & 0.2825 & 1.00 & 0.06 & 0.88 & 1.14 & 0.9905 \\
\hline
\end{tabular}

CHR chromosome, SNP single nucleotide polymorphism, BP base position, $A 1 / A 2$ minor allele/major allele, $F_{-} P$ frequency of minor allele in patients of poor response, $F_{-} G$ frequency of minor allele in patients of good response, $L 95 / U 9595 \%$ Confidence interval, OR odds ratio, $S E$ standard error. 
allele frequency (MAF) of rs1516569 across populations, especially between European (EUR) and East Asian (EAS) populations (Supplementary Fig. 1). The different SNPs between our study and the others may be derived from the sample size. Also a study shows that GRM7 modulates the phosphorylation of cyclic AMP response elementbinding protein (CREB) and the expression of Yesassociated protein (YAP) via direct interaction with $\mathrm{CaM}$, which furtherly adjusts the expression of CyclinD1 and finally influences early cortical development ${ }^{31}$. And the factors affecting early development contribute to the etiology of schizophrenia. In summary, this study furtherly proved GRM7 as the risk factor of schizophrenia.

In terms of antipsychotic drug-efficient pharmacogenetics studies, we found the limited sample recruitment in these studies. The small-sample-set may explain for the inconsistent results of researches on different groups. In our study, nearly 2413 patients were included for exploring the predictive markers. For the sake of determining the perplexing genetic factors involved in the efficacy of antipsychotic treatment, SNPs of GRM7 gene participated in synaptic transmission and plasticity ${ }^{32}$ have been explored for seven common antipsychotic drugs, which uncover the mechanism of antipsychotic response concerning the specified drugs. In general, our study is a correlation analysis between genetic variants and the efficacy of olanzapine, risperidone, quetiapine, aripiprazole, ziprasidone, and haloperidol or perphenazine in the Chinese Schizophrenia.

Our research indicated that rs9883258 in GRM7 was correlated with antipsychotic response which is an intron variant and overlaps 10 transcripts. The 1000 Genomes Project Phase 3 database (http://grch37.ensembl.org) showed that there are significant differences in the minor allele frequency (MAF) of rs9883258 across populations, especially between European (EUR) and East Asian (EAS) populations (Supplementary Fig. 2). The rs779746, rs480409 in GRM7 were associated with olanzapine response, the rs779746 is an intron variant and overlaps 12 transcripts, the rs480409 overlaps 11 transcripts which was in line with the previous CATIE trial ${ }^{33}$; The rs78137319 was associated with treatment response of quetiapine which is a synonymous variant and overlaps 11 transcripts. The SNP rs1154370 was associated with treatment response of risperidone and haloperidol which is an intron variant overlaps 11 transcripts. The SNP rs779746 was associated with treatment response of perphenazine which is an intron variant and overlaps 12 transcripts. However, there are different GRM7 SNPs in antipsychotic response for Schizophrenia patients. the investigation illustrated that rs2133450 in GRM7 was significantly involved in Risperidone response ${ }^{13}$; The association between rs2069062, rs1532544 in GRM7 and the response to lurasidone was determined in the research concerning European and African Ancestries ${ }^{34}$.
At the same time, this research resides in some limitations. Firstly, we did not include the patients who take the other antipsychotic treatment besides the seven drugs, which can narrow the implication of study. Secondly, the samples recruited were the Han Chinese, which will have relatively narrowed significance. Thirdly, we make an associated analysis by using logistic regression under an additive genetic model with our current data, furtherly more comprehensive antipsychotic sample sizes and predictive model may be helpful for more sound results. Although, there are some limitations of the study, our findings that GRM7 was related to antipsychotic treatment efficacy could shed light on interactions between the GRM7 and antipsychotics, which can provide a sound foundation for SCZ therapy.

\section{Acknowledgements \\ This work was supported by grants from the National Key R\&D Program of China (2016YFC1307000), the National Natural Science Foundation of China (81825009, 81571313, 81901358, and 91432304), Natural Science Foundation of Shandong Province (ZR2019BH001), Chinese Academy of Medical Sciences Research Unit (No. 2018RU006) and Peking University Clinical Scientist Program (BMU2019LCKXJ012). We also thank all subjects who participated in our study.}

\begin{abstract}
Author details
${ }^{1}$ Institute of Mental Health, Peking University Sixth Hospital, 100191 Beijing, China. ${ }^{2} \mathrm{NHC}$ Key Laboratory of Mental Health, National Clinical Research Center for Mental Disorders and Key Laboratory of Mental Health, Ministry of Health (Peking University Sixth Hospital), 100191 Beijing, China. ${ }^{3}$ Shandong Collaborative Innovation Center for Diagnosis, Treatment and Behavioral Interventions of Mental Disorders, Department of Psychiatry, Jining Medical University, 272067 Jining, Shandong, China. ${ }^{4}$ PKU-IDG/McGovern Institute for Brain Research, Peking University, 100871 Beijing, China. ${ }^{5}$ Chinese Academy of Medical Sciences Research Unit (No. 2018RU006), Peking University, 100191 Beijing, China
\end{abstract}

Conflict of interest

The authors declare that they have no conflict of interest.

\section{Publisher's note}

Springer Nature remains neutral with regard to jurisdictional claims in published maps and institutional affiliations.

Supplementary Information accompanies this paper at (https://doi.org/ 10.1038/s41398-020-0763-4).

Received: 25 September 2019 Revised: 7 January 2020 Accepted: 12 February 2020

Published online: 03 March 2020

\section{References}

1. Andreasen, N. C. Symptoms, signs, and diagnosis of schizophrenia. Lancet 346, 477-481 (1995).

2. van Os, J., Kenis, G. \& Rutten, B. P. The environment and schizophrenia. Nature 468, 203-212 (2010).

3. Hilker, R. et al. Heritability of schizophrenia and schizophrenia spectrum based on the nationwide Danish twin register. Biol. Psychiatry 83, 492-498 (2018).

4. Wu, Y., Yao, Y. G. \& Luo, X. J. SZDB: a database for schizophrenia genetic research. Schizophr. Bull. 43, 459-471 (2017).

5. Schizophrenia Working Group of the Psychiatric Genomics C. Biological insights from 108 schizophrenia-associated genetic loci. Nature 511, 421-427 (2014). 
6. Carlsson, A., Hansson, L. O., Waters, N. \& Carlsson, M. L. Neurotransmitter aberrations in schizophrenia: new perspectives and therapeutic implications. Life Sci. 61, 75-94 (1997).

7. Shibata, $\mathrm{H}$. et al. Association study of polymorphisms in the group III metabotropic glutamate receptor genes, GRM4 and GRM7, with schizophrenia. Psychiatry Res. 167, 88-96 (2009).

8. Nothen, M. M., Nieratschker, V., Cichon, S. \& Rietschel, M. New findings in the genetics of major psychoses. Dialogues Clin. Neurosci. 12, 85-93 (2010).

9. Jalan-Sakrikar, $\mathrm{N}$. et al. Identification of Positive Allosteric Modulators VU0155094 (ML397) and VU0422288 (ML396) Reveals New Insights into the Biology of Metabotropic Glutamate Receptor 7. ACS Chem. Neurosci. 5, 1221-1237 (2014).

10. Kinoshita, A., Shigemoto, R., Ohishi, H., van der Putten, H. \& Mizuno, N. Immunohistochemical localization of metabotropic glutamate receptors, mGluR7a and mGluR7b, in the central nervous system of the adult rat and mouse: a light and electron microscopic study. J. Comp. Neurol. 393, 332-352 (1998).

11. Kosinski, C. M. et al. Localization of metabotropic glutamate receptor $7 \mathrm{mRNA}$ and mGluR7a protein in the rat basal ganglia. J. Comp. Neurol. 415, 266-284 (1999).

12. Stevenson, J. M. et al. Antipsychotic pharmacogenomics in first episode psychosis: a role for glutamate genes. Transl. Psychiatry 6, e739 (2016).

13. Sacchetti, E. et al. The GRM7 gene, early response to risperidone, and schizophrenia: a genome-wide association study and a confirmatory pharmacogenetic analysis. Pharmacogenomics J. 17, 146-154 (2017).

14. Allen, J. D. \& Bishop, J. R. A systematic review of genome-wide association studies of antipsychotic response. Pharmacogenomics 20 , 291-306 (2019).

15. Arranz, M. J. \& de Leon, J. Pharmacogenetics and pharmacogenomics of schizophrenia: a review of last decade of research. Mol. Psychiatry 12, 707-747 (2007).

16. Yamanouchi, Y. et al. Effect of DRD2, 5-HT2A, and COMT genes on antipsychotic response to risperidone. Pharmacogenomics J. 3, 356-361 (2003).

17. Sullivan, P. F. et al. Genomewide association for schizophrenia in the CATIE study: results of stage 1. Mol. Psychiatry 13, 570-584 (2008).

18. Pardiñas, A. F. et al. Common schizophrenia alleles are enriched in mutationintolerant genes and in regions under strong background selection. Nat. Genet. 50, 381-389 (2018).

19. $\mathrm{Yu}, \mathrm{H}$. et al. Common variants on 2p16.1, 6p22.1 and 10q24.32 are associated with schizophrenia in Han Chinese population. Mol. Psychiatry 22, 954-960 (2017).
20. Lam, M. et al. Comparative genetic architectures of schizophrenia in East Asian and European populations. Nature Genetics 51, 1670-1678 (2019).

21. Pruim, R. J. et al. LocusZoom: regional visualization of genome-wide association scan results. Bioinformatics 26, 2336-2337 (2010).

22. $\mathrm{Yu}, \mathrm{H}$. et al. Five novel loci associated with antipsychotic treatment response in patients with schizophrenia: a genome-wide association study. Lancet Psychiatry 5, 327-338 (2018).

23. Purcell, S. et al. PLINK: a tool set for whole-genome association and population-based linkage analyses. Am. J. Hum. Genet. 81, 559-575 (2007).

24. Ramasamy, A. et al. Genetic variability in the regulation of gene expression in ten regions of the human brain. Nat. Neurosci. 17, 1418-1428 (2014).

25. Jaffe, A. E. et al. Developmental and genetic regulation of the human cortex transcriptome illuminate schizophrenia pathogenesis. Nat. Neurosci. 21, 1117-1125 (2018).

26. Claing, A., Laporte, S. A., Caron, M. G. \& Lefkowitz, R. J. Endocytosis of G proteincoupled receptors: roles of $\mathrm{G}$ protein-coupled receptor kinases and betaarrestin proteins. Prog. Neurobiol. 66, 61-79 (2002).

27. Bray, N. J. et al. No evidence for association between a non-synonymous polymorphism in the gene encoding human metabotropic glutamate receptor 7 and schizophrenia. Psychiatr. Genet. 10, 83-86 (2000).

28. Bolonna, A. A., Kerwin, R. W., Munro, J., Arranz, M. J. \& Makoff, A. J. Polymorphisms in the genes for mGluR types 7 and 8: association studies with schizophrenia. Schizophrenia Res. 47, 99-103 (2001).

29. Chen, X., Long, F., Cai, B., Chen, X. \& Chen, G. A novel relationship for schizophrenia, bipolar and major depressive disorder Part 3: Evidence from chromosome 3 high density association screen. J. Comp. Neurol. 526, 59-79 (2018).

30. Li, W. et al. Significant association of GRM7 and GRM8 genes with schizophrenia and major depressive disorder in the Han Chinese population. Eur. Neuropsychopharmacol. 26, 136-146 (2016).

31. Xia, W., Liu, Y. \& Jiao, J. GRM7 regulates embryonic neurogenesis via CREB and YAP. Stem Cell Rep. 4, 795-810 (2015).

32. Fisher, N. M., Seto, M., Lindsley, C. W. \& Niswender, C. M. Metabotropic glutamate receptor 7: a new therapeutic target in neurodevelopmental disorders. Front. Mol. Neurosci. 11, 387 (2018).

33. Need, A. C. et al. Pharmacogenetics of antipsychotic response in the CATIE trial: a candidate gene analysis. Eur. J. Hum. Genet. 17, 946-957 (2009).

34. Li, J., Yoshikawa, A., Brennan, M. D., Ramsey, T. L. \& Meltzer, H. Y. Genetic predictors of antipsychotic response to lurasidone identified in a genome wide association study and by schizophrenia risk genes. Schizophr. Res. 192, 194-204 (2018) 